\title{
Two-stage Surgery for Endovascular Repair and Laparoscopic Colectomy for a Patient with Abdominal Aortic Aneurysm and Concomitant Colon Cancer: Report of a Case
}

\author{
Hiroki Tanaka, MD, Naoki Unno, MD, Toshio Nakamura, MD, Kiyotaka Kurachi, MD, Naoto Yamamoto, MD, \\ Kazunori Inuzuka, MD, Daisuke Sagara, MD, Minoru Suzuki, MD, Motohiro Nishiyama, MD, and Hiroyuki Konno, MD
}

\begin{abstract}
Surgical management of abdominal aortic aneurysm (AAA) with concomitant malignancy remains controversial. Commercial availability of a stentgraft may change the treatment strategy for such patients. We present a case of AAA with concomitant colon cancer, in which two-stage surgery consisting of EVAR and subsequent laparoscopic colectomy was performed with an interval of six days. The patient's postoperative course was uneventful. For high-risk patients, application of endovascular AAA repair and laparoscopic surgery may decrease the risk of surgical morbidity and mortality.
\end{abstract}

Key words: abdominal aortic aneurysm, stentgraft, colon cancer, laparoscopic surgery

\section{INTRODUCTION}

$\mathrm{T}$ he treatment strategy for abdominal aortic aneurysm (AAA) with concomitant malignancy has been controversial. Particularly, the selection of either one-stage or two-stage surgery has been discussed in previous reports. $^{1-9)}$ However, recent advances in surgical technique have facilitated less invasive treatment in the era of laparoscopic surgery and endovascular aneurysm repair (EVAR), which prompts us to discuss the strategy further. Here, we report a case of an AAA with colon cancer managed by two-stage surgery consisting of EVAR and subsequent laparoscopic colectomy.

\section{CASE}

A 79-year-old male was referred to our hospital with a

Second Department of Surgery, Hamamatsu University School of Medicine, Hamamatsu, Japan

Received: July 8, 2008 Accepted: January 19, 2009

Address for reprint requests to Naoki Unno, MD: Second Department of Surgery, Hamamatsu University School of Medicine, 1-20-1 Handayama, Higashi-ku, Hamamatsu, Shizuoka 431-3192, Japan

Fax: +81-53-435-2273

E-mail: unno@hama-med.ac.jp diagnosis of concomitant sigmoid colon cancer and AAA. He complained of general fatigue due to anemia. Colonoscopy demonstrated an irregularly elevated lesion involving the sub-circumference in the sigmoid colon, in which biopsy demonstrated tubulovillous adenocarcinoma (Fig. 1). Medical history included smoking, hypertension and asthma. Preoperative computed-tomography (CT) showed a $55 \mathrm{~mm}$ fusiform infrarenal AAA without involvement of the aortic bifurcation, which met the anatomical criteria for EVAR (Fig. 2). There were no liver or lung metastases.

The pulmonary functional test showed forced expiratory volume in one second (FEV1.0) of 1.0L and FEV1.0\% of $38 \%$, which was categorized as severe COPD (chronic obstructive pulmonary disease). Considering his age as well as respiratory condition, we chose to perform two-stage surgery consisting of EVAR followed by laparoscopic colectomy. EVAR was performed first under general anesthesia. With a bilateral inguinal incision, a Zenith Endovascular stentgraft ${ }^{\circledR}$ (Cook Ink, Bloomington, USA) was deployed successfully with blood loss of $10 \mathrm{ml}$ and surgical duration of 147 minutes (Fig. 3). The postoperative course was uneventful. He started walking and oral intake on postoperative day 1 .

Six days after EVAR, laparoscopic assisted sigmoidectomy was performed with blood loss of $100 \mathrm{ml}$ and surgi- 

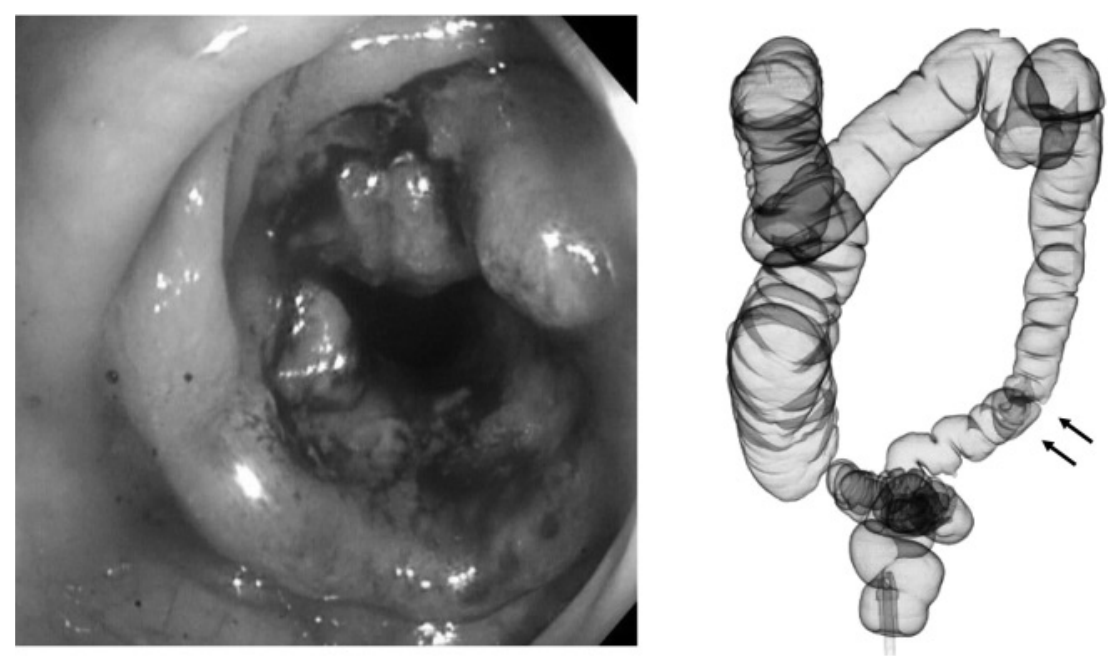

$$
\text { A } \mid \text { B }
$$

Fig. 1

A: Colonoscopy demonstrated an irregularly elevated lesion involving a sub-circumference of the sigmoid colon.

B: Preoperative computed-tomography (CT) colonoscopy. Arrow shows the cancer-induced stenotic region of the sigmoid colon.
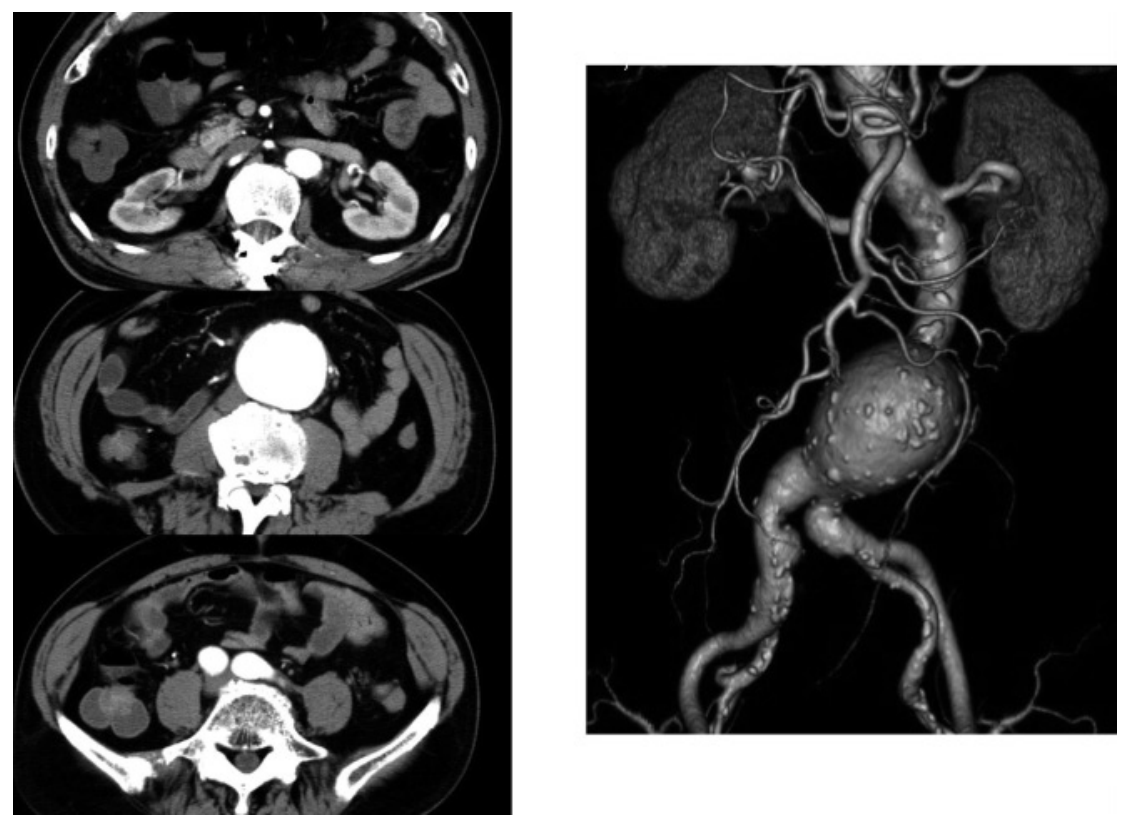

Fig. 2 Preoperative computed-tomography (CT) showed a fusiform infrarenal abdominal aortic aneurysm without involvement of the aortic bifurcation.

cal duration of 144 minutes. The histological examination of the sigmoid colon lesion confirmed a pT3N1 adenocarinoma, which was beyond the serosal layer without penetrating the peritoneum. The resected margins were free of malignancy. D3 lymph node dissection was preformed sufficiently. Therefore, the operation was considered curative. The postoperative course after colectomy was also uneventful.

The patient recovered fully and was discharged on the 16th day after the second operation.

\section{DiscUSSION}

Incidence of AAA concomitant with malignancies is increasing due to the aging population and change of diet in Japan. ${ }^{1-3)}$ For treating such patients, the advantages/ disadvantages of one-/two-stage operation, or malignancy first/AAA first strategy have been discussed. Recent advances in operative procedure and perioperative management enabled the decrease in operative mortality and morbidity in either AAA or malignancy surgery, so that the case of one-stage operation is increasing, which can 

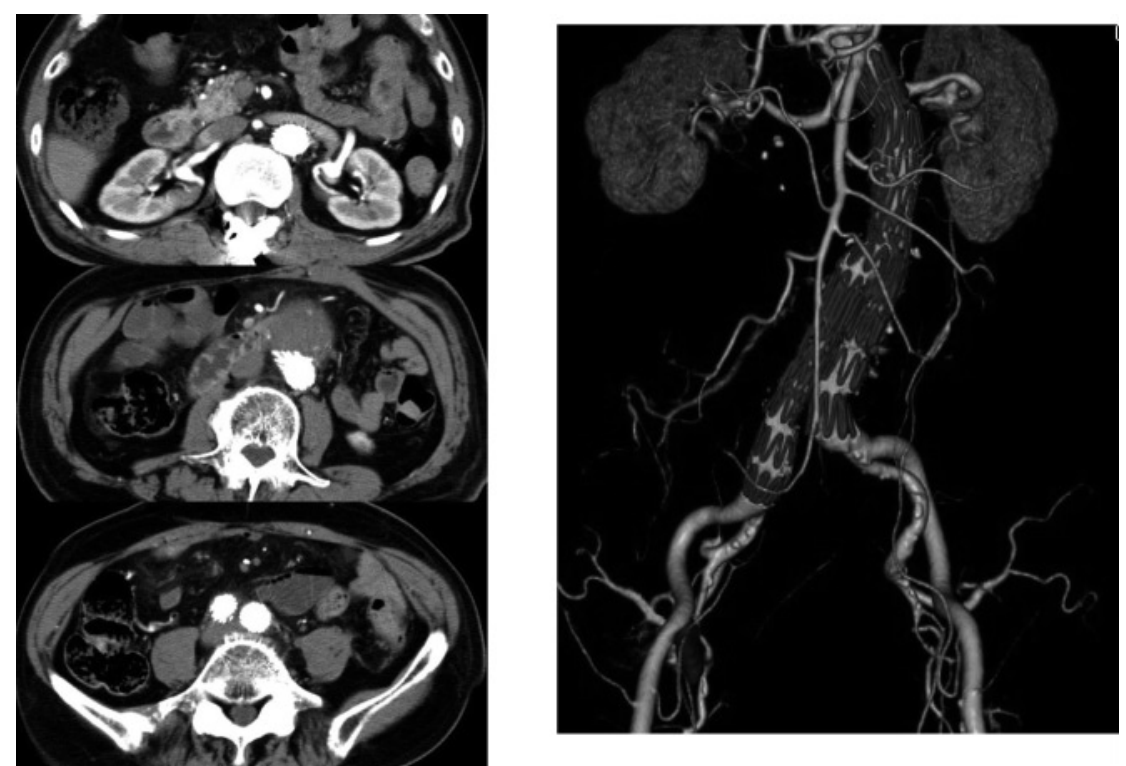

Fig. 3 Postoperative computed-tomography (CT) showed no endoleak.

avoid the risks of either tumor progression or AAA rupture, or the adhesion problem. ${ }^{5-7)}$ On the other hand, a strategy of two-stage operation with AAA repair first, colorectal operation second has been recommended because one-stage operation for both AAA and colorectal cancer may increase the risk of bacterial contamination to the prosthetic graft. ${ }^{8,9)}$

With the advances in surgical techniques for both AAA and malignancy, the discussion regarding the strategy should be renewed. In January 2007, commercial stentgraft (Zenith Endovascular stentgraft ${ }^{\mathrm{B}}$ ) was first approved for re-inversement for EVAR under the Japanese Social Health Insurance System, when the era of EVAR started in Japan. EVAR is a minimally invasive technique without laparotomy, which allows patients to be discharged from hospital within a couple of days postoperatively, so that subsequent operations for malignancy can be performed without delay. Furthermore, EVAR does not increase the difficulties in resecting the malignancy. Further research is necessary to support the EVAR first, malignancy second strategy, or facilitate the rationale for a one-stage procedure to manage both AAA and colon cancer simultaneously.

In this case, we considered the patient to have a high risk for one-stage operation because of his age and respiratory condition (i.e., severe COPD). The longer operative time and/or prolonged general anesthesia might increase the risk of post-operative respiratory failure. Therefore, we chose a two-stage operation with EVAR first and lap- aroscopic sigmoidectomy second with an interval of 6 days when the patient looked to be fully recovered from the first EVAR operation. In Japan, the Social Health Insurance System indicated that a commercial stentgraft should be used for patients for whom open AAA surgery is not the first option. However, the superiority and/or suitability of EVAR compared to open repair for cases with concomitant malignancies has not yet been established. To date, there was only one study comparing the both methods for such patients, in which the outcome of EVAR and open repair of AAA was retrospectively assessed in twenty-five patients undergoing curative treatment of concomitant malignancies. ${ }^{10)}$ They reported a lower morbidity and mortality in EVAR than open repair, but recommended performing another study in a larger series to definitely conclude the superiority of EVAR. Therefore, a prospective and randomized control study should be performed to determine the superiority of EVAR compared to open surgery as well as clarifying whether one stage or two-stage strategy would be optimal for patients with AAA and concomitant malignancy.

\section{REFERENCES}

1) Konno H, Kaneko H, Hachiya T, Maruo Y, Tanaka T, Suzuki S, et al. Surgical management for a malignancy of the digestive organs accompanied with an abdominal aortic aneurysm. Surg Today. 1998; 28 : 988-91. 
2) Kurata S, Nawata K, Hongo H, Suto R, Nagashima H, Kuroda Y, et al. Surgery for abdominal aortic aneurysms associated with malignancy. Surg Today. 1998; 28: 895-9.

3) Komori K, Okadome K, Itoh H, Funahashi S, Sugimachi K. Management of concomitant abdominal aortic aneurysm and gastrointestinal malignancy. Am J Surg. 1993; 166: 108-11.

4) Matsumoto K, Nakamaru M, Obara H, Hayashi S, Harada H, Kitajima M, et al. Surgical strategy for abdominal aortic aneurysm with concurrent symptomatic malignancy. World J Surg. 1999; 23: 248-51.

5) Matsumoto K, Murayama T, Nagasaki K, Osumi K, Tanaka K, Nakamaru M, et al. One-stage surgical management of concomitant abdominal aortic aneurysm and gastric or colorectal cancer. World J Surg. 2002; 26: 434-7.

6) Shimada Y, Sogawa M, Okada A, Namura O, Hayashi $\mathrm{J}$, Iiai $\mathrm{T}$, et al. A single-stage operation for abdominal aortic aneurysm with concomitant colorectal carcino- ma. Ann Thorac Cardiovasc Surg. 2005; 11: 339-42.

7) Illuminati G, Calio' FG, D’Urso A, Lorusso R, Ceccanei G, Vietri F. Simultaneous repair of abdominal aortic aneurysm and resection of unexpected, associated abdominal malignancies. J Surg Oncol. 2004; 88: 234-9.

8) Rivolta N, Piffaretti G, Tozzi M, Lomazzi C, Riva F, Alunno A, et al. Management of simultaneous abdominal aortic aneurysm and colorectal cancer: the rationale of mini-invasive approach. Surg Oncol. 2007; 16 (Suppl 1): S165-7.

9) Baxter NN, Noel AA, Cherry K, Wolff BG. Management of patients with colorectal cancer and concomitant abdominal aortic aneurysm. Dis Colon Rectum. 2002; 45: 165-70.

10) Porcellini M, Nastro P, Bracale U, Brearley S, Giordano P. Endovascular versus open surgical repair of abdominal aortic aneurysm with concomitant malignancy. J Vasc Surg. 2007; 46: 16-23. 